\title{
Pendularité à longue distance
}

\author{
La vitesse des transports comme facteur structurant de l'urbain
}

\section{Introduction: une forme de pendularité en expansion}

En Suisse, la pendularité à longue distance reste rare même si elle est en forte croissance. Elle touche essentiellement des personnes de statut socio-professionnel élevé et se trouve fortement orientée sur l'usage des transports publics. Ce phénomène, qui fait éclater les bassins d'emplois traditionnels, peut être considéré comme une expression du processus de métropolisation [1]. II a été rendu possible par la conjugaison de l'accroissement des vitesses de déplacement des différents systèmes de transport et d'une plus grande acceptation de longs trajets [2].

La «philosophie» sous-jacente au développement des grandes infrastructures rapides (autoroutes, S-Bahn p.ex.) a été la volonté de libérer la population des contraintes de l'espace-temps dans le cadre de leurs déplacements professionnels et de loisirs et d'offrir aux entreprises un gain de productivité. Force est cependant de constater que le pendulaire n'utilise pas les gains de temps offerts pour minimiser ses durées de déplacements; au contraire, il en profite pour réaliser une pendularité plus lointaine. La périurbanisation en est une illustration parfaite.

En claire, le type de rationalité attribué aux usagers dans la planification des transports est une pré-notion erronée qui a eu des conséquences non désirées sur l'évolution des structures urbaines:

- L'étalement urbain. Dans un premier temps, la réalisation de tronçons autoroutiers intervilles ef l'amélioration des réseaux routiers principaux à destination des villes-centre dans les années 60 et 70 ont contribué à renforcer les structures urbaines existantes tout en permettant un étalement et une dédensification des agglomérations, ce qu'atteste la croissance spatiale des agglomérations urbaines entre les recensements fédéraux de 1970, 1980 et 1990.

- La pendularité de longue distance. Dans les années 80, l'émergence de systèmes de transports en réseaux est illustré par la philosophie de Rail 2000 dont les prémices ont vu le jour avec I'horaire cadencé. Par ailleurs, durant la même période, les autoroutes sont devenues un véritable réseau grâce à la liaison de tronçons jusqu'ici isolés (p.ex. par la construction de FribourgLausanne, Lucerne-Olten ou le Gothard). Ceci a été un élément-clé du processus de mise en réseau du tissu urbain helvétique. Les grandes agglomérations ont vu leur centralité et leur marché de l'emploi renforcés.

Ces deux aspects entraînent progressivement la saturation des réseaux par l'accroissement du trafic qu'ils induisent. Cette situation et ses conséquences environnementales nourrissent de nouvelles réflexions sur l'avenir des transports en Suisse. Parmi ces réflexions émergent de grands projets tels que Rail 2000 ou Swissmetro.

\section{Trois hypothèses}

Quels sont les impacts d'un accroissement des vitesses de déplacement? Tester et discuter cette question à travers l'exemple de la pendularité de longue distance sera le fil rouge de cet article. Pour ce faire, nous nous appuyons sur trois hypothèses de base:

Hypothèse 1: La logique sous-jacente aux pratiques modales des pendulaires de longue distance $n^{\prime}$ est pas strictement la minimisation des durées de déplacement.

La plupart des recherches menées sur le comportement des pendulaires concernent les déplacements internes aux agglomérations. Ces recherches démontrent que les navetteurs qui disposent $d$ 'un réel choix modal ne font usage des transports publics que lorsque le stationnement au lieu de travail est difficile et/ou le déplacement est plus court en transports publics [3]. Par ailleurs, elles mettent en évidence l'importance du «désir différentiel» d'utilisation de l'automobile et des transports publics comme facteur explicatif des pratiques modales: I'usage de la voiture est généralement préféré à celui des transports collectifs à cause de la privatisation et de l'individualisation du déplacement qu'il procure à son conducteur [4].

Pour les déplacements de longue distance, notre hypothèse revient à postuler que la préférence d'utilisation à durée de déplacement comparable s'inverse au profit du train: même lorsque I'utilisation du train nécessite une durée de déplacement supérieure, sa part modale est plus forte que celle de la voiture. Cette hypothèse s'appuie sur un triple constat. D'une part, le train direct ou intercity reste un moyen de transport «prestigieux» (particulièrement en première classe) et qu'à ce titre il est désirable. D'autre part, la fatigue induite par la conduite en autoroute sur de longues distances rend pénible l'usage de l'automobile pour de tels déplacements. Enfin, la durée de trajet en train est utilisable pour d'autres activités et qu'à ce titre, même si elle est plus longue, elle ne constitue pas une perte de temps. Ce dernier aspect est particulièrement valable pour les personnes de statut socioprofessionnel élevé qui représentent une part importante des pendulaires de longue distance [5].

Hypothèse 2: Les efforts d'amélioration des vitesses de déplacement sur les réseaux routier et ferroviaire ont un impact sur les flux pendulaires. Nous posons l'hypothèse que des changements dans la relation des durées de déplacement en train et en automobile ont un impact direct sur la répartition modale. La modification de la structure des origines et destinations des déplacements pendulaires joue un rôle central dans ce processus.

Ces réflexions reposent sur deux éléments distincts: les modes de vie et la planification territoriale. Au niveau des modes de vie, elles supposent que la suburbanisation et la périurbanisation résidentielle touchent également les ménages de pendulaires de longue distance. Le standing de certains quartiers et sans doute l'attrait d'un mode de vie «californien» sous-tendraient ce constat. La suburbanisation des emplois est telle qu'elle annule les gains de temps occasionnés par l'amélioration de la vitesse des trains.

Précisons que la vérification de cette hypothèse renvoie directement à la problématique de la ségrégation spatiale croissante dans les grandes agglomérations. Les emplois de cadres ou à forte valeur ajoutée sont concernés par la 
pendularité longue distance, le marché du travail pour les positions inférieures restant largement interne aux agglomérations. Or les origines et destinations du migrateur quotidien ainsi que son choix modal dépendent de la position sociale des répondants.

Hypothèse 3: La mise en service d'un transport public très rapide entre les principaux centres urbains de Suisse a pour impact de renforcer la pendularité inter-agglomération de longue distance aujourd'hui impossible à cause des contraintes de l'espace-temps.

Tester cette hypothèse revient de facto à comparer l'effet multiplicateur des mouvements pendulaires en fonction des gains de temps.

$\mathrm{Si}$ elle se vérifie, nous pourrons conclure qu'une modification des accessibilités relatives entre villes, par la réalisation de transports publics à grande vitesse, contribue à accroître la hiérarchie entre agglomérations en renforçant la centralité d'un ou de plusieurs grands centres urbains. Cela signifierait également que la conjecture de Zahavi [6] concernant les budgets-temps intervient aussi comme mécanisme régulateur des mouvements pendulaires.

Les enjeux qui sous-tendent ces trois hypothèses sont considérables. Ils se situent à plusieurs niveaux:

- Discuter les conditions de concurrence entre moyens de transports signifie tout d'abord tester les fondements $d^{\prime}$ une conception de la planification des transports basée sur le postulat d'une minimisation des durées de déplacement qui est aujourd'hui encore invoquée pour justifier des investissements colossaux.

- Lier mobilité quotidienne, mobilité résidentielle et migration suppose par ailleurs proposer une approche intégrée entre «l'habiter» et «le circuler» qui fait totalement défaut actuellement, tant pour des questions de frontières disciplinaires qu'à cause de la complexité des processus que cette analyse suppose. $\mathrm{Si}$ les hypothèses confirment la nécessité d'une telle approche, cela contribuera à ouvrir la porte à un véritable urbanisme des réseaux.

- Réfléchir aux impacts territoriaux d'un affranchissement progressif des contraintes de l'espace-temps par un accroissement des vitesses de déplacement entre certaines grandes agglomérations suppose une réflexion sur l'avenir du système urbain suisse sous l'angle des inégalités spatiales et des questions de centralités. Le test des hypothèses permettra d'approfondir quelques pistes de réflexion sur ce thème [7].

\section{Méthodologie}

Pour tester les hypothèses énoncées, nous proposons une approche qui se réfère à des couples de relations entre grands centres. Il s'agit des inter-relations entre Genève et Lausanne (et viceversa) ainsi qu'entre Zurich et Berne (également dans les deux sens). Le choix de ces couples d'agglomérations est fondé sur quatre facteurs: leur taille et leur importance qui engendrent depuis longtemps des flux pendulaires inter-agglomérations suffisamment importants pour pouvoir être comparés, leur niveau de centralité à peu près équivalent, la situation dans les deux régions linguistiques principales $d u$ pays - facteur souvent considéré comme explicatif de comportements différenciés en matière de mobilité - et la position sur la dorsale ouest-est. Pour ces quatre pôles, les évolutions entre 1970 et 1990 sont observées sur la base de la définition 1990 des agglomérations.

Le recensement de la population est la source la plus riche en ce qui concerne la documentation des flux quotidiens de la population [8]. Bien qu'il ne tienne compte que d'un seul type de déplacement, à savoir la pendularité, ces données permettent - grâce à leur exhaustivité - la calibration des modèles de transport [9].

En ce qui concerne le développement des infrastructures des routes et du rail et leur desserte, la documentation annuelle de l'Office fédéral des routes [10] ainsi que les horaires des transports publics [1 11] permettent de dresser une comparaison diachronique.

A l'aide de ces informations, nous avons procédé aux calculs suivants pour les relations entre les deux paires $d^{\prime}$ agglomérations et en distinguant les liens entre deux villes-centre, entre une ville- centre et une couronne [12] (et inversément) ainsi qu'entre deux couronnes: a) Part modale des transports publics pour les flux pendulaires sur les relations étudiées: Pourcentage des utilisateurs des transports publics (en principe le train) par rapport à l'ensemble des personnes actives ayant indiqué leur moyen de transport [13].

b) Comparaison des durées de déplacement en automobile et en transports publics. Ces durées ont été calculées sur la base des méthodes suivantes:

Durée des trajets par la route: la vitesse moyenne sur l'autoroute est estimée à $100 \mathrm{~km} / \mathrm{h}$, sur une route cantonale à $70 \mathrm{~km} / \mathrm{h}$ et à l'intérieur des agglomérations à $40 \mathrm{~km} / \mathrm{h}$. S'y ajoute un temps de quatre minutes pour le stationnement.

Durée des trajets en transports publics: la démarche de calcul pour les trajets en transports publics contient les trajets à pied et en bus et les temps de correspondance à la gare dans la région d'habitation, le temps du trajet en train, le temps de correspondance à la gare d'arrivée et le temps de bus/train et de marche jusqu'au travail.

Les calculs décrits ont été réalisés sur la base d'hypothèses concernant les localisations d'habitation et d'emploi dans les villes-centre et les couronnes de chaque agglomération et cela pour les trois recensements.

\section{Analyse de l'évolution de l'offre et de la demande}

\subsection{L'évolution des flux pendulaires entre les agglomérations}

Pour amorcer l'analyse, le tableau 1 reprend les totaux des quatre grands tableaux traitant des comparaisons de durées de trajets, du nombre de pendulaires et des répartitions modales (tableaux 2 à 5).

Ce tableau montre d'emblée trois effets distincts:

a) L'effet de la distance. L'intensité entre les deux centres lémaniques, plus proches, est nettement plus élevée que les échanges entre Berne et Zurich.

b) La très forte croissance de la pendularité. Partant d'un niveau très bas, 
voire insignifiant en 1970, la pendularité interville a très fortement augmenté et concerne actuellement près de 400 personnes à Zurich, 750 à Berne, 2000 à Genève et 4500 à Lausanne.

c) La hiérarchie urbaine. Les chiffres relatifs constituent un indicateur de centralité: Genève et Zurich exercent une attirance nettement plus forte que leur ville partenaire. Ce phénomène est à mettre en relation avec un marché de l'emploi plus large et plus différencié dans le centre le plus important. II en découle que dans ces agglomérations les niveaux de salaire et de loyer sont plus élevés.

\subsection{L'évolution de l'offre des transports routier et ferroviaire}

Les deux premières colonnes des tableaux 2 à 5 présentent les durées de déplacement en train et par la route et leur évolution depuis 1970.

Les noyaux des agglomérations de Berne et Zurich se trouvent à une distance de $120 \mathrm{~km}$ environ. L'autoroute $\mathrm{N}$ 1, l'axe dorsal du réseau autoroutier suisse, a été construite dans les années 60. Le tronçon entre Berne et Lenzbourg a été inauguré en 1967. Le prolongement vers Baden (1970) et Zurich-Hardturm (1971) avait été réalisé trop tard pour avoir un effet $d$ 'incitation sur les pendulaires lors du recensement de 1970. Les autres tronçons autoroutiers dans l'agglomération de Berne ont été ouverts au cours des années 70 , tandis que le contournement au nord de la ville de Zurich (Weiningen-Glattbrugg et Milchbucktunnel) ne date que de 1985; le contournement sud-ouest de la ville n'est pas encore réalisé.

La construction de deux nouveaux tracés ferroviaires sur la ligne ZurichBerne a permis d'abaisser sensiblement la durée du trajet entre ces deux pôles. En 1975, la construction du tunnel du Heitersberg a apporté un gain de 9 minutes. Dans les années 80 , I'ouverture du tunnel du Born (1982) ainsi que $d^{\prime}$ autres mesures ont rapporté un gain supplémentaire de 10 minutes, réduisant ainsi la durée à 71 minutes [14]. En ce qui concerne les trajets menant dans la couronne des agglomérations,

\begin{tabular}{|c|c|c|c|c|c|c|}
\hline \multirow[b]{2}{*}{ Liaison } & \multicolumn{3}{|c|}{ en chiffres absolus } & \multicolumn{3}{|c|}{$\begin{array}{l}\text { en pourcent des actifs } \\
\text { de l'agglomération } \\
\text { d'habitation }\end{array}$} \\
\hline & 1970 & 1980 & 1990 & 1970 & 1980 & 1990 \\
\hline Berne-Zurich & 63 & 268 & 745 & 0,04 & 0,17 & 0,42 \\
\hline Zurich-Berne & 33 & 147 & 383 & 0,01 & 0,03 & 0,07 \\
\hline Lausanne-Genève & 1146 & 1857 & 4489 & 0,90 & 1,43 & 2,92 \\
\hline Genève-Lausanne & 549 & 994 & 1996 & 0,30 & 0,52 & 0,90 \\
\hline
\end{tabular}

Tableau 1: Pendulaires en chiffres absolus et par rapport au nombre de personnes actives, entre 1970 et 1990

\begin{tabular}{lccccc}
\hline & $\begin{array}{c}\text { train } \\
\text { (min) }\end{array}$ & $\begin{array}{l}\text { auto } \\
\text { (min) }\end{array}$ & $\begin{array}{l}\text { rel. de } \\
\text { durée }\end{array}$ & $\begin{array}{r}\text { nombre } \\
\text { pendul. }\end{array}$ & $\begin{array}{l}\% \text { transp. } \\
\text { publ. }\end{array}$ \\
\hline ville/ville 70 & 129 & 123 & 1,05 & 31 & $(81)$ \\
ville/ville 80 & 122 & 100 & 1,22 & 90 & 78 \\
ville/ville 90 & 112 & 100 & 1,12 & 224 & 78 \\
& 152 & 128 & 1,19 & 3 & \\
ville/couronne 70 & 145 & 105 & 1,38 & 34 & \multicolumn{1}{c}{$(50)$} \\
ville/couronne 80 & 126 & 99 & 1,27 & 114 & 65 \\
ville/couronne 90 & & & & & \\
& 146 & 115 & 1,27 & 26 & $(69)$ \\
couronne/ville 70 & 144 & 97 & 1,48 & 106 & 67 \\
couronne/ville 80 & 125 & 97 & 1,29 & 211 & 77 \\
couronne/ville 90 & & & & & \\
& 169 & 120 & 1,41 & 3 &. \\
couronne/couronne 70 & 167 & 102 & 1,64 & 38 & $(26)$ \\
couronne/couronne 80 & 167 \\
couronne/couronne 90 & 139 & 96 & 1,48 & 196 & 35 \\
& & & & & \\
agglo/agglo 70 & & & & 63 & 73 \\
agglo/agglo 80 & & & & 268 & 63 \\
agglo/agglo 90 & & & & 734 & 66
\end{tabular}

Tableau 2: Durée et relation de durée des trajets, nombre de pendulaires et répartition modale, de 1970 à 1990 - Berne-Zurich

\begin{tabular}{lccccc}
\hline & $\begin{array}{c}\text { train } \\
\text { (min) }\end{array}$ & $\begin{array}{l}\text { auto } \\
(\mathrm{min})\end{array}$ & $\begin{array}{l}\text { rel. de } \\
\text { durée }\end{array}$ & $\begin{array}{r}\text { nombre } \\
\text { pendul. }\end{array}$ & $\begin{array}{l}\text { \% transp. } \\
\text { publ. }\end{array}$ \\
\hline ville/ville 70 & 128 & 128 & 1,02 & 26 & $(81)$ \\
ville/ville 80 & 121 & 103 & 1,17 & 65 & 74 \\
ville/ville 90 & 111 & 103 & 1,08 & 128 & 84 \\
ville/couronne 70 & 148 & 121 & 1,22 & 3 &. \\
ville/couronne 80 & 145 & 103 & 1,41 & 6 & $(67)$ \\
ville/couronne 90 & 126 & 97 & 1,30 & 28 & $(50)$ \\
couronne/ville 70 & 149 & 125 & 1,19 & 4 &. \\
couronne/ville 80 & 142 & 103 & 1,38 & 67 & 51 \\
couronne/ville 90 & 123 & 103 & 1,19 & 152 & 56 \\
& & & & & \\
couronne/couronne 70 & 169 & 121 & 1,40 & 1 &. \\
couronne/couronne 80 & 166 & 103 & 1,61 & 9 &. \\
couronne/couronne 90 & 138 & 97 & 1,42 & 75 & 36 \\
& & & & & \\
agglo/agglo 70 & & & & 34 & $(76)$ \\
agglo/agglo 80 & & & & 147 & 61 \\
agglo/agglo 90 & & & & 378 & 61 \\
\hline
\end{tabular}

Tableau 3: Durée et relation de durée des trajets, nombre de pendulaires et répartition modale, de 1970 à 1990 - Zurich-Berne 
I'introduction de l'horaire cadencé en 1982 a apporté un gain décisif, puisque les temps de correspondance ont été réduits de moitié environ.

Les deux villes du bord du Léman se trouvent distantes de $60 \mathrm{~km}$. L'autoroute qui les relie a été la première construite en Suisse (1964). Le contournement de Lausanne a été inauguré en 1974, celui de Genève en 1987 (jusqu'à l'aéroport) et en 1993 (jusqu'à Bardonnex).

Depuis les années 50, des trains directs circulent à une cadence horaire. Le temps de trajet est resté quasiment inchangé depuis 1970 (34 et 33 minutes).

Contrairement aux relations entre Berne et Zurich, I'offre routière et ferroviaire et par conséquent la relation de durée sont restées stables (si on ne tient pas compte des effets de saturation des réseaux routiers).

\subsection{L'évolution de la répartition modale} L'examen des répartitions modales entre les deux couples d'agglomérations étudiés montre que la part du train est globalement plus forte que celle de l'automobile, malgré le fait que le train $s^{\prime}$ avère plus lent sur toutes les relations observées. Ce constat confirme notre première hypothèse selon laquelle le train est compétitif sur des déplacements de longue distance.

Si la part modale du train a néanmoins diminué, c'est essentiellement dû à une modification des relations originedestination ce qui tend à confirmer notre seconde hypothèse (cf. 4.5). Par ailleurs, l'examen de détail de la répartition modale et de l'intensité des flux amène quelques commentaires complémentaires (cf. tableaux 2 à 5 , totaux entre agglomérations).

Les trajets entre Zurich et Berne étaient encore très faibles en 1970. La durée des trajets dépassait largement les deux heures. A l'époque, le trajet let son prix) excerçait une sélection sociale maximale - seuls les emplois les plus spécialisés fonctionnaient sur un modèle de ce type lqui aujourd'hui est peut-être comparable aux liaisons par avion entre Genève et Zurich, empruntées par 55 pendulaires en 1990).

\begin{tabular}{lccccc}
\hline & $\begin{array}{c}\text { train } \\
\text { (min) }\end{array}$ & $\begin{array}{l}\text { auto } \\
(\mathrm{min})\end{array}$ & $\begin{array}{l}\text { rel. de } \\
\text { durée }\end{array}$ & $\begin{array}{r}\text { nombre } \\
\text { pendul. }\end{array}$ & $\begin{array}{l}\% \text { transp. } \\
\text { publ. }\end{array}$ \\
\hline ville/ville 70 & 74 & 63 & 1,17 & 508 & 81 \\
ville/ville 80 & 73 & 63 & 1,16 & 642 & 83 \\
ville/ville 90 & 73 & 63 & 1,16 & 1143 & 86 \\
ville/couronne 70 & 83 & 63 & 1,32 & 129 & 49 \\
ville/couronne 80 & 82 & 63 & 1,30 & 192 & 46 \\
ville/couronne 90 & 82 & 60 & 1,37 & 612 & 56 \\
& & & & & \\
couronne/ville 70 & 97 & 63 & 1,54 & 372 & 65 \\
couronne/ville 80 & 82 & 63 & 1,30 & 693 & 61 \\
couronne/ville 90 & 81 & 63 & 1,28 & 1493 & 68 \\
& & & & & \\
couronne/couronne 70 & 106 & 63 & 1,68 & 137 & 36 \\
couronne/couronne 80 & 91 & 63 & 1,44 & 330 & 36 \\
couronne/couronne 90 & 90 & 60 & 1,50 & 1241 & 34 \\
& & & & & \\
agglo/agglo 70 & & & & 1146 & $(76)$ \\
agglo/agglo 80 & & & & 1857 & 63 \\
agglo/agglo 90 & & & & 4489 & 62 \\
\hline
\end{tabular}

Tableau 4: Durée et relation de durée des trajets, nombre de pendulaires et répartition modale, de 1970 à 1990 Lausanne-Genève

\begin{tabular}{lccccc}
\hline & $\begin{array}{c}\text { train } \\
\text { (min) }\end{array}$ & $\begin{array}{l}\text { auto } \\
(\mathrm{min})\end{array}$ & $\begin{array}{l}\text { rel. de } \\
\text { durée }\end{array}$ & $\begin{array}{c}\text { nombre } \\
\text { pendul. }\end{array}$ & $\begin{array}{l}\% \text { transp. } \\
\text { publ. }\end{array}$ \\
\hline $\begin{array}{l}\text { ville/ville 70 } \\
\text { ville/ville 80 }\end{array}$ & 73 & 63 & 1,16 & 139 & 81 \\
ville/ville 90 & 72 & 63 & 1,14 & 182 & 75 \\
& 72 & 60 & 1,20 & 338 & 74 \\
ville/couronne 70 & 99 & 62 & 1,60 & 39 & $(23)$ \\
ville/couronne 80 & 84 & 62 & 1,35 & 72 & 53 \\
ville/couronne 90 & 83 & 59 & 1,41 & 153 & 43 \\
& & & & & \\
couronne/ville 70 & 85 & 64 & 1,33 & 267 & 66 \\
couronne/ville 80 & 83 & 64 & 1,30 & 502 & 71 \\
couronne/ville 90 & 83 & 61 & 1,36 & 811 & 65 \\
& & & & & \\
couronne/couronne 70 & 111 & 63 & 1,76 & 104 & 44 \\
couronne/couronne 80 & 95 & 63 & 1,51 & 238 & 32 \\
couronne/couronne 90 & 94 & 60 & 1,57 & 694 & 20 \\
& & & & & \\
agglo/agglo 70 & & & & 549 & 59 \\
agglo/agglo 80 & & & & 994 & 51 \\
agglo/agglo 90 & & & & 1996 & 45 \\
\hline
\end{tabular}

Tableau 5: Durée et relation de durée des trajets, nombre de pendulaires et répartition modale, de 1970 à 1990 -

Genève-Lausanne

Entre 1970 et 1980, on observe une diminution sensible du temps de trajet par la route, tandis que le gain par le rail reste faible. Cette évolution occasionne une diminution de la proportion des utilisateurs du train, même si - en chiffres absolus - la pendularité augmente fortement. Au cours de la décennie suivante, le gain de temps par la route n'est qu'insignifiant (il concerne pourtant l'accès au pôle d'emploi du Glattal à proximité de l'aéroport de Kloten). Par contre, une accélération des trajets par le train et l'introduction de I'horaire cadencé (1982) rendent les transports publics plus compétitifs, notamment pour les liaisons vers les banlieves. La part modale du train aug- 


\begin{tabular}{|c|c|c|c|c|}
\hline \multirow{2}{*}{$\begin{array}{l}\text { Relation entre agglomérations } \\
\text { Type d'origine-destination } \\
\text { Berne-Zurich, ville/ville }\end{array}$} & \multicolumn{2}{|c|}{$\begin{array}{l}\% \text { pendulaires } \\
\text { attendus eff. } \\
\text { en } 1980\end{array}$} & \multicolumn{2}{|c|}{$\begin{array}{l}\% \text { pendulaires } \\
\text { attendus eff. } \\
\text { en } 1990\end{array}$} \\
\hline & 27,8 & 33,6 & 22,9 & 30,1 \\
\hline Berne-Zurich, ville/couronne & 18,5 & 12,7 & 18,4 & 15,3 \\
\hline Berne-Zurich, couronne/ville & 32,3 & 39,6 & 32,6 & 28,3 \\
\hline Berne-Zurich, couronne/couronne & 21,4 & 14,2 & 26,1 & 26,3 \\
\hline Zurich-Berne, ville/ville & 29,5 & 44,2 & 25,7 & 33,4 \\
\hline Zurich-Berne, ville/couronne & 11,7 & 4,2 & 12,5 & 7,3 \\
\hline Zurich-Berne, couronne/ville & 42,2 & 45,6 & 41,6 & 39,7 \\
\hline Zurich-Berne, couronne/couronne & 16,7 & 6,1 & 20,1 & 19,6 \\
\hline Lausanne-Genève, ville/ville & 29,9 & 34,6 & 24,9 & 25,5 \\
\hline Lausanne-Genève, ville/couronne & 17,4 & 10,3 & 17,7 & 13,5 \\
\hline Lausanne-Genève, couronne/ville & 33,2 & 37,3 & 33,5 & 33,3 \\
\hline Lausanne-Genève, couronne/couronne & 19,4 & 17,8 & 23,9 & 27,6 \\
\hline Genève-Lausanne, ville/ville & 25,9 & 18,3 & 21,9 & 16,9 \\
\hline Genève-Lausanne, ville/couronne & 17,1 & 7,2 & 19,1 & 7,7 \\
\hline Genève-Lausanne, couronne/ville & 34,4 & 50,5 & 31,5 & 40,6 \\
\hline Genève-Lausanne, couronne/couronne & 22,7 & 23,9 & 27,5 & 34,8 \\
\hline
\end{tabular}

Tableau 6: Proportion de pendulaires attendus et observés selon les types d'originedestination, en 1980 et en 1990

mente à nouveau (de Berne à Zurich de $63 \%$ à $66 \%$, dans le sens inverse il reste à $61 \%$ ) et ceci pour tous les types de relations origine-destination. En 1990, la pendularité interville entre les deux agglomérations est dix fois plus élevée que vingt ans auparavant.

Entre Genève et Lausanne, la distance réelle et la distance-temps correspondent à peu près à la moitié de celles entre Berne et Zurich. Les flux pendulaires sont donc nettement plus intenses. Leur nombre a quadruplé au cours de ces 20 dernières années. Conformément au niveau de centralité supérieur de Genève, les flux partant de Lausanne sont deux fois plus importants en chiffres absolus et trois fois plus importants en chiffres relatifs que dans le sens inverse (cette relation est $d^{\prime}$ ailleurs restée inchangée depuis 1970).

Parallèlement à l'augmentation des mouvements pendulaires, la part modale du train a considérablement baissé pour la relation Genève-Lausanne (de $59 \%$ à $45 \%$ ) et plus faiblement dans le sens inverse (de $67 \%$ à $62 \%$ ).

\subsection{L'évolution des origines- destinations des pendulaires}

Les processus de suburbanisation et de périurbanisation des dernières décennies sont caractérisés par la décentralisation relative et absolve des habitants des villes-centre vers les couronnes. La répartition des emplois a suivi la même évolution avec un décalage temporel. En ce qui concerne la pendularité à longue distance, la probabilité qu'un mouvement se fasse de ville-centre à villecentre diminue donc constamment. Les trajets pour lesquels la répartition modale est la plus favorable au train sont donc de moins en moins nombreux. Notre enquête permet de saisir les changements structurels de ces flux, l'évolution des relations de durée entre modes et le comportement des pendulaires.

Dans les quatre agglomérations, à peu près $60 \%$ des actifs habitent en couronne. En ce qui concerne la localisation des emplois, Berne garde cependant une structure spatiale plus fortement centralisée $133 \%$ des emplois sont situés en couronnes à Berne contre $45 \%$ à Zurich et à Lausanne et $42 \%$ à Genève). Il découle de ces structures différenciées que les flux de pendulaires à destination de l'agglomération bernoise sont plus fortement dirigés vers la villecentre que les autres flux étudiés.

En examinant l'évolution entre les durées comparées de trajets automobile/ train et la répartition modale, nous constatons que (cf. tableaux 2 à 5 , troisième et cinquième colonnes, ainsi que le graphique):

- Lorsque les durées de déplacement sont comparables, la part modale du train avoisine les $80 \%$. Entre 1970 et 1990, cette relation a un peu évolué au profit du train [15]. Lorsque les durées sont favorables à l'usage de la voiture, son utilisation est nettement plus forte.

- La répartition modale varie en fonction du type de relation origine-destination. Sur des relations ville-ville, elle est très favorable au train, a contrario elle est fortement orientée sur la voiture pour les relations couronne-couronne. Pour les deux autres types de relation, on observe une situation intermédiaire avec une part du train plus élevée pour les mouvements couronne-centre (ce qui découle de la possibilité de parkings d'échange dans l'agglomération d'habitation et des conditions de stationnement en moyenne plus difficiles au lieu de travail). Cette observation montre que la répartition modale n'est pas le strict reflet de la minimisation des durées de déplacement, ce qui confirme notre première hypothèse.

- Sur chaque type d'origine-destination Zurich-Berne, la part modale du train a baissé entre 1970 et 1980 et augmenté durant la décennie suivante pour retrouver un niveau proche de 1970. Ceci correspond exactement à l'évolution de la qualité de l'offre qui, comme nous l'avons vu au point 4.2, a favorisé la route dans les années 70 et le rail dans les années 80.

Pour les origines-destinations entre Genève et Lausanne, les relations de durée sont restées plus ou moins stables entre 1970 et 1990. II en est de même pour la répartition modale. Si, malgré cela, la part modale globale du train entre ces agglomérations a considérablement baissé (elle est passée de $67 \%$ à $62 \%$ sur Lausanne-Genève et de $59 \%$ à $45 \%$ sur Genève-Lausanne), ceci s'explique d'une part par la déconcentration massive de l'habitat et de l'emploi dans ces agglomérations, ef $d^{\prime}$ autre part par la proximité spatiale des couronnes des deux agglomérations [16].

- Pour affiner l'analyse, nous avons procédé à une comparaison entre les flux pendulaires attendus et les chiffres observés lors des recensements. Les pendulaires attendus sont calculés selon un modèle qui postule une répartition 


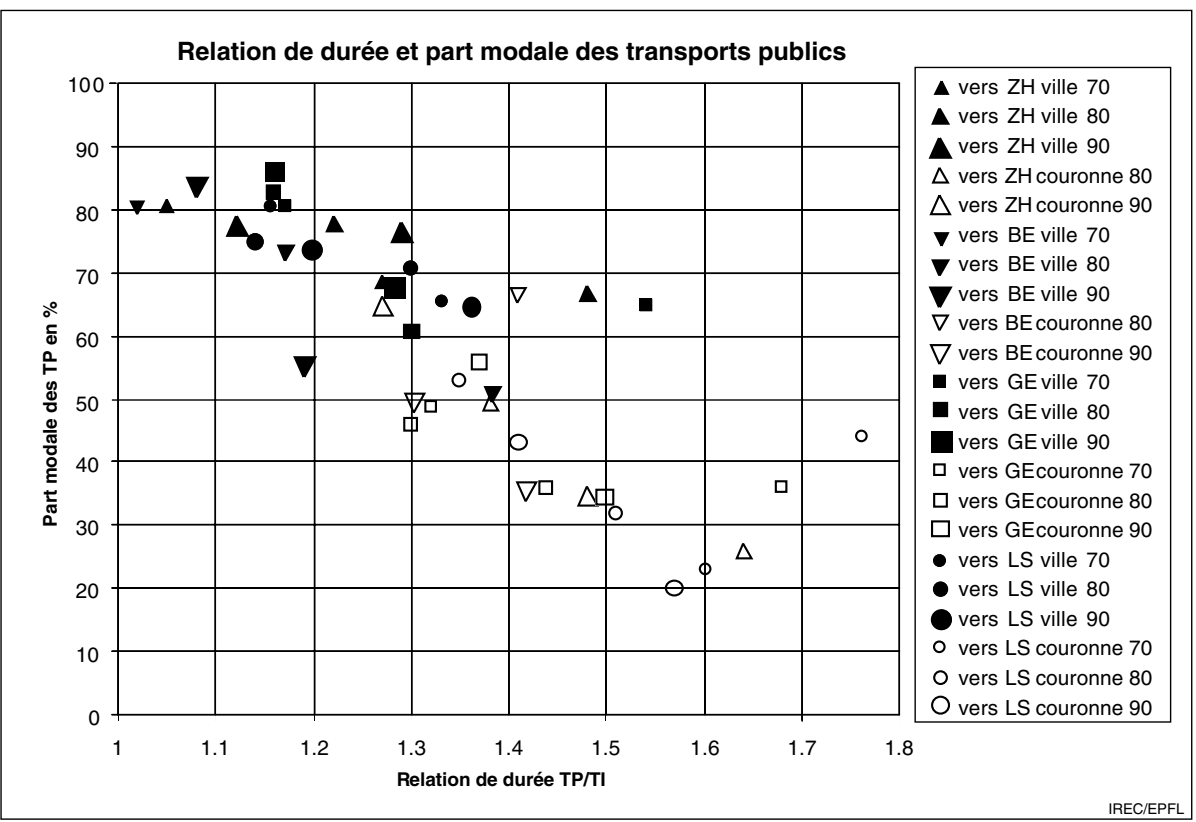

proportionnelle des flux pendulaires origine-destination suivant les localisations résidentielles et des emplois. Les chiffres effectifs montrent une surreprésentation faiblissante des liaisons ville-ville mais également, depuis 1990, une surreprésentation des liaisons couronne-couronne.

Si la surreprésentation ville-ville découle de la performance du train sur ces relations, la surreprésentation de la liaison couronne-couronne provient des durées beaucoup plus courtes en voiture. Une dimension sociale s'y ajoute à cette seconde tendance, à savoir une nouvelle spatialisation des couches sociales supérieures: elles habitent en zone périurbaine et travaillent en zone suburbaine, même si celle-ci se trouve dans une autre agglomération.

\section{Conclusions: la vitesse de trans- port comme facteur structurant}

Cette analyse diachronique sur l'évolution de la pendularité à longue distance a permis une série de constatations. Nous allons les reprendre dans l'esprit de nos hypothèses de départ pour en tirer quelques conclusions en rapport avec les enjeux actuels.

1. Les trajets pendulaires à longue distance sont ceux qui ont augmenté proportionnellement le plus au cours de ces dernières décennies. Ils ont gardé jusqu'ici une forte orientation sur les transports publics, vu les grandes distances à parcourir et la relation de durée relativement favorable au train. $A$ relation de durée égale entre le train et la route, la part des transports publics a même légèrement augmenté.
La comparaison de la pendularité entre les deux paires d'agglomérations choisies montre un niveau plus élevé d'échanges entre les agglomérations lémaniques, ce qui peut être attribué à la distance plus courte qui les sépare. Par contre, à relation de durée comparable entre modes, la part du train est identique entre les quatre agglomérations. Nous pouvons en déduire qu'au niveau de la pendularité à longue distance, il n'y a pas de différences de comportement entre les habitants des deux régions linguistiques. Ceci infirme des préjugés répandus quant aux différences de comportement entre Romands et Alémaniques. La recherche de Kaufmann et Stofer (op. cit.) aboutit à la même conclusion pour la pendularité intraagglomération. La différence d'usage est fonction de l'offre de transport et de la structure spatiale des agglomérations.

2. Si, au niveau global des flux entre agglomérations, la part modale des transports publics diminue, cela s'explique par une modification de la composition des flux en termes d'originedestination et va dans le sens de notre seconde hypothèse: les relations de ville-centre à ville-centre diminuent proportionnellement au détriment des flux centre-couronne et surtout couronne-couronne. Pour ces derniers, la comparaison des durées est très favorable à la voiture (il s'agit de la course la plus rapide), ce qui, malgré de longues distances à parcourir, entraîne une utilisation fréquente de l'automobile (celleci est utilisée dans $65 \%$ des cas environ).
3. Nos analyses concernant l'évolution de la pendularité entre les grands centres laissent entrevoir l'émergence $d$ 'un processus $d$ 'interpénétrations plus fort entre les grandes agglomérations du pays: en faisant allusion aux processus observés à un niveau spatial inférieur, on peut esquisser des tendances futures. En effet, une augmentation forte des pendulaires entre deux agglomérations est souvent un facteur-clé de la croissance démographique de l'agglomération la plus petite. Entre 1980 et 1990, l'augmentation de la population des agglomérations d'Yverdon, de Bienne, de Schaffhouse ou de Wil était due uniquement à l'essor de la pendularité vers des centres plus grands, tandis que la croissance des emplois n'était que très faible, voire négative. En découlent plusieurs conclusions:

- L'augmentation démographique des personnes actives est certes un facteur positif pour ces agglomérations. Vu la composition sociale (élevée) des pendulaires à longue distance, ces agglomérations en tirent un profit économique supplémentaire. (A part des couches moyennes, beaucoup de jeunes de la région font la navette vers les centres plus grands.)

- Ces pendulaires n'ont pas seulement un impact sur la structure démographique, ils induisent aussi de nouvelles structures socio-spatiales dans ces agglomérations: les lieux d'habitation de ces pendulaires sont généralement situés dans les villes-centre, pour des raisons fonctionnelles (durée de trajet) et symboliques (le prestige d'un centre-ville de petite ville). La concentration relative des pendulaires à longue distance sur les centres d'agglomération entraîne des flux de pendulaires dérivés des actifs travaillant sur place: ce sont ces derniers qui, à leur tour, sont obligés de faire la navette entre la couronne et la ville-centre de ces agglomérations secondaires. Ces flux se font majoritairement par transports privés.

- C'est ainsi que le bilan environnemental positif des pendulaires à longue distance (répartition modale très favorable au train) est à nuancer par les conséquences indirectes qu'il engendre sur $d^{\prime}$ autres flux pendulaires. On peut dès 
lors parler - en faisant allusion au comportement de migration - de chaînes de pendularité.

4. Concernant l'armature urbaine du pays, les pendulaires à longue distance jouent un rôle important dans les interrelations toujours plus fortes entre les agglomérations. Vu le statut socio-professionnel plutôt élevé de ces navetteurs, ceux-ci contribuent à une répartition plus équilibrée des personnes actives dans l'espace et notamment à une "gentrification» des villes-centre secondaires. A contrario, une concentration plus forte des emplois à qualification élevée dans les grands centres et leurs couronnes appauvrit le marché de l'emploi des agglomérations secondaires au détriment des grands centres et leurs couronnes. Ce processus s'effectue à travers un transfert des emplois les plus qualifiés des villes secondaires vers les grands centres, mais surtout à cause du choix prioritaire par des nouvelles implantations dans les grandes agglomérations.

5. Deux grands projets de transport concernent les transports publics entre les agglomérations. II s'agit de Rail 2000 et de Swissmetro [17]. Entre Zurich et Berne, Rail 2000 prévoit un gain de temps de 15 minutes par rapport à la situation actuelle, pour arriver à 57 minutes. Entre Genève et Lausanne, le gain sera faible. Le projet Swissmetro propose de parcourir le tronçon Genève-Lausanne en 20 minutes (12 minutes de trajet et deux fois 4 minutes supplémentaires d'accès) [18]. Entre Zurich et Berne, les projets actuellement en discussion prévoient un arrêt à Lucerne; il en découlerait un trajet de 40 minutes.

- Les deux projets auraient un impact sur les flux pendulaires. Rail 2000 va dynamiser les tendances actuelles et donc augmenter le nombre de pendulaires à longue distance et la part modale du train. Swissmetro impliquerait, par contre, un changement conceptuel profond. Son impact dépendrait de la philosophie sous-jacente du projet: serat-il conçu comme un métro «urbain», soit un transport public de masse ou, au contraire, sera-til conçu comme une substitution de l'avion? Les prix du tra- jet, la fréquence, l'accessibilité et la qualité du voyage, mais aussi l'emplacement des stations par rapport aux systèmes des transports urbains existants, sont les paramètres qui permettront de répondre à cette question. En cas de réalisation de Swissmetro, l'évolution du système urbain suisse dépendrait de la philosophie retenue.

- La réalisation de Swissmetro réduirait la distance-temps entre grands centres à celle séparant aujourd'hui ces centres de leurs agglomérations satellites. Se pose donc la question de savoir, si les plus petites des grandes agglomérations (Berne, Lausanne, mais également Bâle) seraient rétrogradées à la fonction d'agglomérations satellites ou bien si elles pourront maintenir leur poids relatif à travers la spécialisation économique jusqu'à maintenant toujours assez forte.

- Une solution de Swissmetro «populaire» renforcerait certainement l'accroissement de la dépendance hiérarchique entre les grandes agglomérations; l'élargissement des bassins serait donc profitable aux pôles les plus forts. Un Swissmetro à fonction élitaire, par contre, changerait probablement moins cette hiérarchie. Dans les deux cas, le point faible de ce projet est qu'il concerne des flux pendulaires déjà les plus favorables aux transports publics.

\section{Remarques et références}

[1] Leresche Jean-Philippe, Dominique Joye et Michel Bassand, Métropolisations, Georg, Genève, 1995.

[2] Cf. Salomon Ilan, Piet Bovy et Jean-Pierre Orfeuil (éds.), A billion trips a day, Tradition and Transition in European Travel Patterns, Kluwer Academic Publishers, 1993. Ils notent une évolution très similaire dans tous les pays d'Europe de l'Ouest. Trois tendances la caractérisent:

- Le nombre moyen de déplacements reste très stable. II s'établit généralement entre 2,5 et 3 déplacements par personne et par jour. La structure des déplacements par motifs se modifie lentement: la part de la pendularité diminue (de moins en moins de personnes rentrent chez elles à midi) au profit des déplacements de loisirs qui tendent à se développer.
- La durée quotidienne totale consacrée aux déplacements reste stable, confirmant ainsi au niveau global la conjecture de Zahavi. Cette durée moyenne s'établit aux alentours d'une heure de déplacements quotidiens par personne et par jour.

- Les distances parcourues quotidiennement ont crû depuis les années 60 et elles s'établissent entre 25 et 35 kilomètres parcourus par personne et par jour selon les pays.

Ces moyennes cachent sans doute des différences très nettes selon le type de pendularité. En particulier l'émergence de la pendularité de longue distance pèse lourd dans l'accroissement des distances parcourues par personne et par jour depuis les années 60 .

[3] Cf. Kaufmann Vincent et Suzanne Stofer, Le report modal de l'automobile vers les transports publics, Rapport de recherche IREC no 126, Lausanne, 1995. Cette recherche porte sur une comparaison des logiques sous-jacentes aux pratiques modales dans les agglomérations de Berne, Genève et Lausanne. Elle montre que lorsque les transports publics sont plus rapides que I'utilisation de l'automobile pour des déplacements pendulaires, ceux-ci sont utilisés dans seulement $50 \%$ des cas dans les deux agglomérations romandes contre $83 \%$ des cas dans l'agglomération bernoise. Ceci s'explique par les différences de conditions de stationnement à destination (taux de stationnement réservé beaucoup plus faible au lieu de travail à Berne) et la qualité de l'offre de transports publics plus élevée dans cette dernière agglomération (moins de changements de lignes, offre ferroviaire plus dense). [4] Yonnet Paul, Jeux, modes et masses, Gallimard, Paris, 1985.

Brög Werner, Les motifs psychologiques qui guident les usagers, Table ronde de la CEMT no 34, Paris, 1977.

[5] Massot Marie-Hélène, Espaces de vie, espaces de travail: 15 ans d'évolution, INRETS, Arcueil, juillet 1995.

[6] Zahavi Y., The UMOT project, USDOT, Washington, 1979.

[7] Jemelin Christophe, Pour une nouvelle cartographie des flux, Université de Lausanne, 1995. L'auteur relève que les principaux flux pendulaires dans le canton de Vaud entre les recensements de 1970, 1980 et 1990 montrent une dépendance croissante des petites agglomérations.

[8] A la suite du dénombrement de 1990, les résultats des recensements antérieurs de 1970 et de 1980 ont pu être harmonisées de manière telle qu'on dispose actuellement d'une série qui couvre l'évolution de la pendularité sur 20 ans, c'est-à-dire les matrices complètes entre les 3021 communes suisses y compris les caractéristiques démographiques et socio-professionnelles. Le comportement des pendulaires (moyens de transport, durées des trajets) est également connu. 
[9] Les résultats du RFP de 1990 concernant la pendularité ont fait l'objet de plusieurs recherches, cf.

- Dozio Alessandro, La mobilité quotidienne dans les cinq grandes agglomérations suisses, Office fédéral de la statistique, Berne, 1995.

- Dozio Alessandro, La mobilité quotidienne dans l'agglomération lausannoise, OESES, Lausanne, décembre 1994.

- SCRIS, STT, SAT, TRANSITEC, Déplacements des pendulaires dans le canton de Vaud: 20 régions sous la loupe, Lausanne, janvier 1995.

- Gächter Ernst et Daniel Aegerter, Eidgenössische Volkszählung 1990, Pendlerund Arbeitsplatzstatistik für die Stadt Bern, Bern, April 1995.

- Amt für Statistik des Kts. Luzern, Pendlerströme und Pendlerprofile, Eidg. Volkszählung 1990, Luzern, 1994.

[10] Routes nationales suisses, INFO, publication annuelle, Office fédéral des routes, Berne.

[11] CFF, Indicateur officiel, éditions 1970, 1980 et 1990.

[12] Pour les origines et destinations situées en couronnes, une sélection d'une dizaine de communes a été opérée pour représenter chaque agglomération.

[13] La statistique des pendulaires contient un certain nombre d'actifs qui disent utiliser des moyens de transport apparemment erroné (à pied ou en tram de Zurich à Berne, p.ex.). Visiblement, les tests de plausibilité n'ont pas toujours été appliqués correctement. En 1990, de tels cas sont relativement nombreux pour les personnes actives ayant deux domiciles et, par conséquent, deux types de comportement pendulaire, à savoir celui entre le domicile "économique» et la place de travail au cours de la semaine et celui du retour aux domicile «légal» le weekend. Ces personnes ont été exclues de nos calculs.

[14] Depuis 1996, le trajet s'est réduit à 70 minutes; lors de la finition du projet Rail 2000 avec notamment la construction du tronçon entre Mattstetten et Rothrist, le temps de trajet devrait être de 57 minutes.

[15] Le graphique montre l'ensemble des relations origine-destination entre 1970 et 1990 pour les flux d'au moins 50 personnes. Pour une relation de durée de 1,1, la part modale des transports publics était de $80 \%$ en 1970 , de $83 \%$ en 1980 et de $85 \%$ en 1990. Les coefficients de régression (R2) entre la relation de durée et la part modale du train sont passés de 0,63 en 1970 à 0,65 en 1980 et à 0,81 en 1990 .

[16] Morges et Nyon, qui font partie des agglomérations de Lausanne et Genève, disposent chacune d'un arrêt de train direct. Leur distance $\mathrm{n}^{\prime}$ excède pas $28 \mathrm{~km}$, leurs inter-relations ne peuvent donc être consi- dérées comme de la pendularité à longue distance. En supprimant le district de Nyon de nos calculs, la part modale entre Genève et Lausanne est effectivement modifiée: part modale du train Nyon-agglomération lausannoise $52 \% \quad(1970), \quad 39 \% \quad(1980), 35 \%$ (1990); agglomération genevoise sans la ville-centre-agglomération lausannoise $61 \%$ (1970), 58\% (1980), 44\% (1990).

[17] Cf. Perret Francis Luc, Jean-Pierre Baumgartner et Jean-Daniel Favrod, Swissmetro, partie I, Analyse prévisionnelle de la demande de transport, Département fédéral des transports, des communications et de I'énergie, ITEP, Lausanne, avril 1990; Ecole polytechnique fédérale de Lausanne, Swissmetro, Synthèse de l'étude préliminaire, janvier-mars 1993, ainsi que Hutter René, Die Raumverträglichkeit der Swissmetro, ORLBerichte 94, ETH Zürich, vdf, 1995.

[18] La liaison Genève-Lausanne est désignée comme tronçon pilote, cf. I'article paru dans «Le Nouveau Quotidien» le 7 mai 1996. 